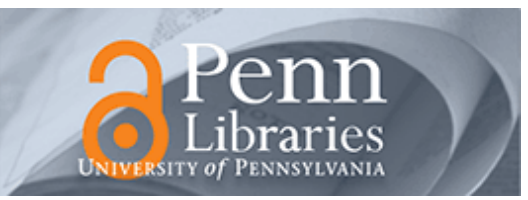

University of Pennsylvania

ScholarlyCommons

May 2008

\title{
Gravimetric chemical sensor based on the direct integration of SWNTS on ALN Contour-Mode MEMS resonators
}

\author{
Matteo Rinaldi \\ University of Pennsylvania, rinaldim@seas.upenn.edu \\ Chiara Zuniga \\ University of Pennsylvania, zunigac@seas.upenn.edu \\ Nipun Sinha \\ University of Pennsylvania \\ Marzie Taheri \\ University of Pennsylvania \\ Samuel M. Khamis

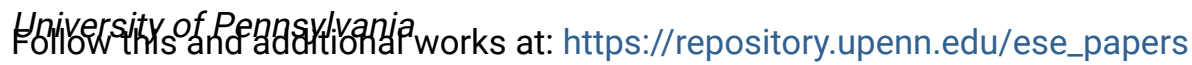 \\ Part of the Electrical and Electronics Commons
}

See next page for additional authors

\section{Recommended Citation}

Matteo Rinaldi, Chiara Zuniga, Nipun Sinha, Marzie Taheri, Samuel M. Khamis, Alan T. Johnson, and Gianluca Piazza, "Gravimetric chemical sensor based on the direct integration of SWNTS on ALN ContourMode MEMS resonators", , 443-448. May 2008.

Copyright 2008 IEEE. Reprinted from 2008 IEEE International Frequency Control, May 2008, pages 443-448.

This material is posted here with permission of the IEEE. Such permission of the IEEE does not in any way imply IEEE endorsement of any of the University of Pennsylvania's products or services. Internal or personal use of this material is permitted. However, permission to reprint/republish this material for advertising or promotional purposes or for creating new collective works for resale or redistribution must be obtained from the IEEE by writing to pubs-permissions@ieee.org. By choosing to view this document, you agree to all provisions of the copyright laws protecting it.

This paper is posted at ScholarlyCommons. https://repository.upenn.edu/ese_papers/457

For more information, please contact repository@pobox.upenn.edu. 


\title{
Gravimetric chemical sensor based on the direct integration of SWNTS on ALN Contour-Mode MEMS resonators
}

\author{
Abstract \\ This paper reports on the first demonstration of a gravimetric chemical sensor based on direct integration \\ of Single Wall Carbon Nanotubes (SWNTs) grown by Chemical Vapor Deposition (CVD) on AIN Contour- \\ Mode MicroElectroMechanical (MEMS) resonators. In this first prototype the ability of SWNTs to readily \\ adsorb volatile organic chemicals has been combined with the capability of AIN Contour-Mode MEMS \\ resonator to provide for different levels of sensitivity due to separate frequencies of operation on the \\ same die. Two devices with resonance frequencies of $287 \mathrm{MHz}$ and $442 \mathrm{MHz}$ have been exposed to \\ different concentrations of DMMP in the range from 80 to $800 \mathrm{ppm}$. Values of mass sensitivity equal to \\ $1.8 \mathrm{KHz} / \mathrm{pg}$ and $2.65 \mathrm{KHz} / \mathrm{pg}$ respectively have been measured.

\section{Keywords} \\ gravimetric sensors, carbon nanotubes, AIN contour-mode resonators, DMMP

\section{Disciplines} \\ Electrical and Electronics

\section{Comments} \\ Copyright 2008 IEEE. Reprinted from 2008 IEEE International Frequency Control, May 2008, pages \\ 443-448. \\ This material is posted here with permission of the IEEE. Such permission of the IEEE does not in any way \\ imply IEEE endorsement of any of the University of Pennsylvania's products or services. Internal or \\ personal use of this material is permitted. However, permission to reprint/republish this material for \\ advertising or promotional purposes or for creating new collective works for resale or redistribution must \\ be obtained from the IEEE by writing to pubs-permissions@ieee.org. By choosing to view this document, \\ you agree to all provisions of the copyright laws protecting it.
}

\section{Author(s)}

Matteo Rinaldi, Chiara Zuniga, Nipun Sinha, Marzie Taheri, Samuel M. Khamis, Alan T. Johnson, and Gianluca Piazza 


\section{Gravimetric Chemical Sensor based on the Direct Integration of SWNTs on AlN Contour-Mode MEMS Resonators}

\author{
Matteo Rinaldi ${ }^{a, *}$, Chiara Zuniga ${ }^{a, *}$, Nipun Sinha ${ }^{b}$, \\ Marzie Taheri ${ }^{a}$ and Gianluca Piazza ${ }^{a}$ \\ ${ }^{a}$ Department of Electrical \& Systems Engineering \\ ${ }^{b}$ Department of Mechanical Engineering and Appl. Mechanics \\ University of Pennsylvania \\ Philadelphia, US \\ rinaldim@seas.upenn.edu ,zunigac@seas.upenn.edu
}

\author{
Samuel M. Khamis and A.T. Johnson \\ Department of Physics and Astronomy \\ University of Pennsylvania \\ Philadelphia, US
}

\begin{abstract}
This paper reports on the first demonstration of a gravimetric chemical sensor based on direct integration of Single Wall Carbon Nanotubes (SWNTs) grown by Chemical Vapor Deposition (CVD) on AIN Contour-Mode MicroElectroMechanical (MEMS) resonators. In this first prototype the ability of SWNTs to readily adsorb volatile organic chemicals has been combined with the capability of AIN Contour-Mode MEMS resonator to provide for different levels of sensitivity due to separate frequencies of operation on the same die. Two devices with resonance frequencies of $287 \mathrm{MHz}$ and $442 \mathrm{MHz}$ have been exposed to different concentrations of DMMP in the range from 80 to $800 \mathrm{ppm}$. Values of mass sensitivity equal to $1.8 \mathrm{KHz} / \mathrm{pg}$ and $2.65 \mathrm{KHz} / \mathrm{pg}$ respectively have been measured.
\end{abstract}

\section{INTRODUCTION}

In recent years lots of efforts have been dedicated towards the fabrication of micromachined resonant devices for high performance chemical sensing. In particular the demand of miniaturized smart sensors for detection of multiple hazardous volatile organic chemicals or warfare agents has been growing. The crucial parameters for this kind of VOC detectors are sensitivity, limit of detection and response time, which depend on the resonance frequency and the properties of the chemically active sensitive layer. Moreover, size, portability, ease of monitoring and cost are other fundamental requirements for real-life environment sensors.

Under this point of view, Quartz Crystal Microbalances (QCM) have successfully been used in many bio-chemical applications [1]. Their extremely high frequency stability allows the detection of very small frequency shifts providing limits of mass detection in the order of few nanograms. Nevertheless, it turns out that the mass sensitivity of QCMs is heavily limited by their relatively low frequency of operation (few tens of $\mathrm{MHz}$ ); this does not permit the use of these devices for the detection of very small quantities of volatile organic chemicals or warfare agents. Moreover, when the fabrication of smart, compact, portable and low cost electronic noses for multiple gas detection is required, QCMs fail to represent a good solution because of their large dimensions and their inability to be directly integrated on silicon.

In this perspective RF MEMS/NEMS devices, such as cantilever beams [2] and thin film bulk acoustic resonators [3], have been showing great potentialities as gravimetric sensors. Thanks to their reduced size, which provides them with higher frequencies of operation than QCM devices, they can achieve unprecedented limit of detection in the atto or zepto gram range. On the other hand, the quality factor of MEMS/NEMS resonators is lower than the one achievable with quartz crystals; hence, their frequency stability is also lower. Nevertheless, the mass sensitivity of a resonator is proportional to the square of its resonance frequency [4]; therefore, if the increase in the frequency of operation is bigger than the reduction of the quality factor, the larger frequency change per unit of mass can compensate for the increased minimum detectable frequency shift and will result in the ability to resolve smaller changes of the measurand [5].

Although improved limit of mass detection can be achieved with MEMS/NEMS devices, there are still some issues related to their effective use as VOC sensors. For instance, the greatly reduced size of nano-cantilever beams limits the area dedicated to the sensitive layer, therefore significantly decreasing the amount of mass adsorbed on the beam surface given a certain concentration of the analyte in the environment. This reduction in adsorbed mass can actually limit the improved mass sensitivity and reduce the global sensitivity and limit of detection to the analyte concentration. On the other hand, even if thin film bulk

* These authors contributed equally to this work. 
acoustic resonators (FBARs) can guarantee high frequency of operation, without significantly reducing the area available for the adsorbing layer, they cannot provide for multiple frequencies of operations on the same chip; this factor ultimately limits their employment for the fabrication of single chip electronic noses.

In this paper, Aluminum Nitride (AlN) Contour-Mode resonators first reported by Piazza et al [6] are investigated as novel and high performance gravimetric sensors for VOC detection. Since the resonance frequency of this kind of devices is lithographically set, multiple frequencies are easily achievable on the same chip. Moreover, high frequency of operation, high quality factor in air $(1,000-3,000)$ and low motional resistance have been demonstrated in AlN ContourMode resonators [6], therefore making these devices suitable for the fabrication of compact, low cost and wireless sensor platforms. Piezoelectrically-transduced contour-mode resonant mass sensors have been previously demonstrated [78] but only for low frequencies of operation (tens of $\mathrm{MHz}$ ) and using a conventional parylene-based adsorbtion layer. Instead, in this work, the capability to achieve multiple and higher frequencies of operation (and therefore sensitivities) is shown using a surface enhancing sensitive layer based on Single Wall Carbon Nanotubes (SWNTs).

From the previous discussion, it can be inferred that in order to improve the performance of gravimetric sensors for multiple VOCs detection, it is not only necessary to increase their frequency of operation, but it is also essential to deal with the chemical/physical properties of the sensitive layer responsible for the adsorbtion process. SWNTs have been showing great potentials for chemical sensing [9-10]. In particular, they offer some advantages as adsorbing layer with respect to polymeric films. Thanks to their unique surface to volume ratio, SWNTs provide the device with a large number of adsorbing sites. At the same time, their high acoustic impedance offers a better acoustic match to the resonator and potentially lowers damping. The deposition of nanocomposite films as sensitive layers on piezoelectrically-transduced thin film bulk acoustic resonators has been previously demonstrated [3]. Differently, in this paper, the direct growth of high purity SWNTs by Chemical Vapor Deposition (therefore using a mass manufacturing process) is shown for the first time.

\section{SENSOR DESIGN}

The sensitivity to mass loading for a higher order contour mode resonator (Fig. 1) can be expressed through the Sauerbrey equation [4]:

$$
\frac{\Delta f}{\Delta m}=-\frac{2 f_{0}^{2}}{n \cdot A \cdot \sqrt{E_{0} \cdot \rho_{0}}}=\frac{S_{0}}{n}
$$

where $f_{0}$ is the operating frequency of the resonator; $n$ is the number of sub-resonators in parallel that forms the multifinger device; $A$ is the cross-sectional area; and $E_{0}$ and $\rho_{0}$ are Young's modulus and mass density of the composite stack, respectively. Its evaluation represents the starting point towards the sensor design optimization.
As previously reported [6], the resonance frequency of a width-extensional higher order contour-mode resonator (Fig.1) is approximately set by the width, $W$, of a single finger and the material properties, $E_{0}$ and $\rho_{0}$ as shown in equation (2).

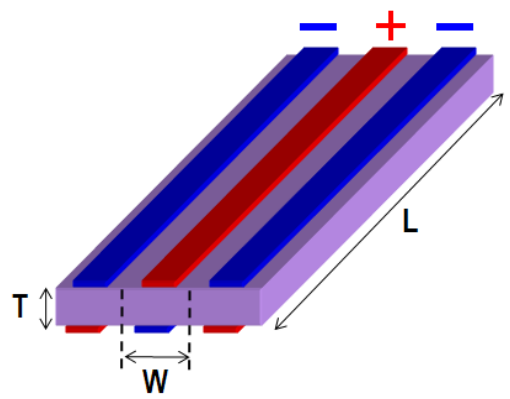

Figure 1. Schematic representation of a 3 fingers higher order AlN contour mode resonator.

$$
f_{0}=\frac{1}{2 W} \sqrt{\frac{E_{0}}{\rho_{0}}}
$$

The application of a vertical electric field across the device thickness induces an in plane dilatation of the structure through the $d_{31}$ AlN piezoelectric coefficient. However, the induced displacement of the resonator is not uniform over its entire width since the mode-shape of vibration obeys to a harmonic motion that can be approximately described as:

$$
u(x)=B \cdot \sin \left(\frac{\pi}{W} x\right)
$$

where $B$ is a constant describing the maximum vibration amplitude and $u(x)$ is the displacement along the $x$-direction. The mode-shape of vibration (Fig. 2) clearly shows that the maximum displacement is attained on the lateral surfaces of the device; therefore, at this location the kinetic energy of the system is also at its maximum.

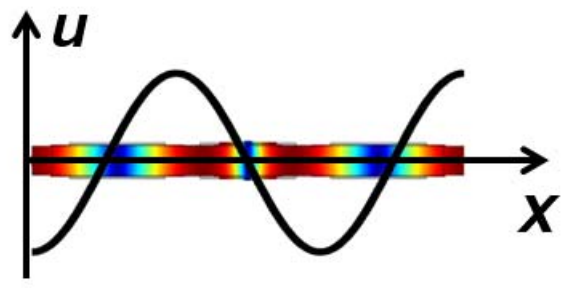

Figure 2. Mode shape of vibration of a 3 fingers resonator obtained via COMSOL FEM simulations: the red regions undergo maximum displacement.

Due to its small mass, the resonance frequency of a MEMS resonator is significantly affected by a slight change in the mass of the device. Since the greatest response to mass loading is achieved when the additional mass induces the greatest perturbation to the system, it can intuitively be understood that the surfaces where the kinetic energy is maximum are the most sensitive to changes in mass. As already stated, the regions associated with maximum kinetic 
energy are the sidewalls; unfortunately, they are hardly accessible for the deposition of a sensitive layer and limited in area for contour-mode resonators.

However, a close analysis of the mode-shape of vibration expression can suggest the idea to dedicate the top surface of the resonator to the deposition of the sensing layer. Because only few monolayers of the analyte are generally absorbed on the surface of the resonator, we can assume that no significant changes to device stiffness occur, but the adsorbed mass transfers completely into a mass change. There is also a significant portion of the contour-mode resonator top area that moves with large velocity. The fact that the loading mass is not distributed where the kinetic energy is maximum can be taken into account by computing the equivalent mass at that point and using that value of mass for the sensitivity calculation:

$$
M_{e q}=\rho \cdot S \cdot \int_{-W / 2}^{W / 2} \sin ^{2}\left(\frac{\pi}{W} \cdot x\right) \cdot d x=\frac{M_{a d d}}{2}
$$

where $S$ and $\rho$ are the cross-sectional area and density of the loading layer; $M_{a d d}$ is the amount of adsorbed mass and $M_{e q}$ is its equivalent value at the point of maximum velocity. Although only half of the loading mass is actually sensed by the device, there is a net gain in the area available for sensing. This gain is equal to the ratio between the top surface and sidewalls areas:

$$
\begin{gathered}
A_{\text {sidewalls }}=2 \cdot L \cdot T \\
A_{\text {top }}=W \cdot n \cdot L \\
A_{\text {eff }}=0.5 \cdot \frac{W \cdot n}{2 T} A_{\text {sidewalls }}
\end{gathered}
$$

where $A_{\text {eff }}$ stands for the effective area (i.e. the area coated with the sensing layer). The effective area is a key parameter for a gravimetric gas sensor since it plays a crucial role in the definition of the sensitivity to variations of the analyte concentration in the environment. In fact, the sensitivity to concentration is directly proportional to the effective area:

$$
\frac{\Delta f}{\Delta c} \propto \frac{S_{0}}{n} \cdot A_{e f f}
$$

which, substituting $A_{\text {eff }}$ from (5), can be expressed as:

$$
\frac{\Delta f}{\Delta c} \propto \frac{S_{0}}{n} \cdot 0.5 \cdot \frac{W \cdot n}{2 T} \cdot A_{\text {sidewalls }}=S_{\text {eff }}
$$

Ultimately, the sensitivity of a contour-mode resonator to variations in the analyte concentration is significantly improved by the increased area dedicated to the sensing layer.

The mass sensitivity value $\left(S_{0} / n\right)$ for the fabricated devices was analytically evaluated and found to be $1.8 \mathrm{KHz} / \mathrm{pg}$ for a $287 \mathrm{MHz}$ resonator $(\mathrm{T}=2 \mu \mathrm{m}, \mathrm{L}=200 \mu \mathrm{m}, \mathrm{W}=15 \mu \mathrm{m}, \mathrm{n}=$ 3) and $2.65 \mathrm{KHz} / \mathrm{pg}$ for a $442 \mathrm{MHz}$ resonator $(\mathrm{T}=2 \mu \mathrm{m}, \mathrm{L}=$
$200 \mu \mathrm{m}, \mathrm{W}=10 \mu \mathrm{m}, \mathrm{n}=5)$. These values are calculated considering that the sensitive layer is uniformly spread over the entire top surface of the resonator. In case the top surface of the resonator is not uniformly covered, then the correction factor calculated in (4) should be evaluated over a smaller spatial interval than the actual width of the sub-resonator.

A 2D Finite Element Method (FEM) analysis has been performed with the aid of COMSOL Multiphysics to validate the obtained analytical values of effective sensitivity $\left(S_{\text {eff }}\right)$. A thin rectangular-shaped mass was added alternatively to the sidewalls and the top surface of the resonator. Then, the device sensitivity was evaluated by gradually varying the density of the added mass and computing the corresponding linear shift in the resonance frequency.

Below, Tables I and II show the comparison between analytical and simulated values of effective sensitivity $\left(S_{\text {eff }}\right)$ for resonators with frequencies of operation close to the ones of the fabricated devices discussed in section IV.

TABLE I. VALUES OF $\mathrm{S}_{\mathrm{EFF}}$ FOR A $289 \mathrm{MHZ}$ RESONATOR

\begin{tabular}{c|c|c}
$289 \mathrm{MHz}$ & Sidewalls & Top Surface \\
\hline Simulated & $2.40 \mathrm{KHz} \cdot \mu \mathrm{m}^{2} / \mathrm{fg}$ & $13.50 \mathrm{KHz} \cdot \mu \mathrm{m}^{2} / \mathrm{fg}$ \\
\hline Analytical & $2.42 \mathrm{KHz} \cdot \mu \mathrm{m}^{2} / \mathrm{fg}$ & $13.65 \mathrm{KHz} \cdot \mu \mathrm{m}^{2} / \mathrm{fg}$
\end{tabular}

TABLE II. VALUES OF $\mathrm{S}_{\text {EFF }}$ FOR A $463 \mathrm{MHZ}$ RESONATOR

\begin{tabular}{c|c|c}
$\mathbf{4 6 3} \mathbf{M H z}$ & Sidewalls & Top Surface \\
\hline Simulated & $4.8 \mathrm{KHz} \cdot \mu \mathrm{m}^{2} / \mathrm{fg}$ & $30 \mathrm{KHz} \cdot \mu \mathrm{m}^{2} / \mathrm{fg}$ \\
\hline Analytical & $4.3 \mathrm{KHz} \cdot \mu \mathrm{m}^{2} / \mathrm{fg}$ & $24.8 \mathrm{KHz} \cdot \mu \mathrm{m}^{2} / \mathrm{fg}$
\end{tabular}

\section{FABRICATION}

A combination of top-down microfabrication techniques for the resonators and bottom-up growth for SWNTs has been employed to realize the nano-enabled microelectromechanical sensors of this work. The resonant devices are fabricated using the same process employed for the Aluminum Nitride (AIN) resonators and filters that have been previously demonstrated by Piazza et al [11]. The nanotube growth is included in this process before resonator release from the $\mathrm{Si}$ substrate by dry isotropic etching in $\mathrm{XeF}_{2}$. After completing the basic steps for the making of the resonator, a wafer is diced in smaller chips so that they can fit in the CVD reactor used for the SWNT growth. Each silicon chip is covered by sputtered $\mathrm{SiO}_{2}$, which acts as seed layer for the nanotubes growth. The growth process is performed by catalytic CVD technique [12]. In this process, an iron catalyst layer is evaporated onto the entire unreleased die. Then the temperature in the CVD chamber is steadily ramped up to the desired growth temperature of $\sim 900{ }^{\circ} \mathrm{C}$. During this process the iron on the surface is reduced to elemental form and its diffusion leads to the formation of nanoscale iron grains that 
act as SWNTs growth sites. Once the desired growth temperature is reached, SWNTs are grown by catalytic decomposition of methane. Polymethylglutarimide resist (PMGI) is then used, instead of standard photoresist, to cover the nanotubes and protect them during the following fabrication steps. PMGI has the advantage of not sticking to the SWNTs and avoids that the SWNTs are stripped off when it is removed. The PMGI is patterned by standard lithographic techniques in such a fashion that only the nanotubes on the top surfaces of the resonators are being protected. During the following dry etching of the oxide seed layer in $\mathrm{CF}_{4}$-based chemistry and the subsequent release step, the nanotubes are removed from everywhere except for the regions protected by PMGI. This fabrication process permits the direct integration of bottom-up grown nanotubes with topdown fabricated resonators. Although, this process has been demonstrated at the die level because the actual dimensions of the CVD reactor cannot accommodate bigger substrates, it can be effectively extended to the wafer level [13] and it is therefore amenable to large scale manufacturing processes.

\section{EXPERIMENTAL RESULTS}

In order to test the fabricated devices, the resonators functionalized by SWNTs were exposed to different concentrations of Dimethyl methylphosphonate (DMMP), a simulant for Sarin nerve agent. The corresponding shifts in resonance frequency due to the different amounts of adsorbed gas molecules were monitored. The measurement setup, the device response and its interpretation are presented in the following sections.

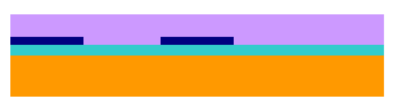

(a)

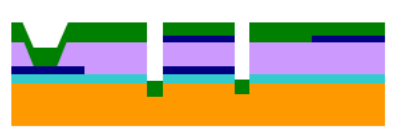

(c)

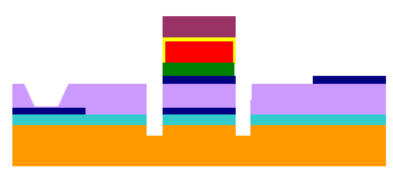

(e)

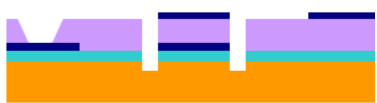

(b)

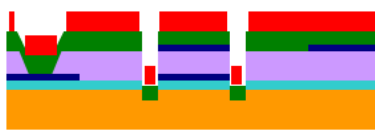

(d)

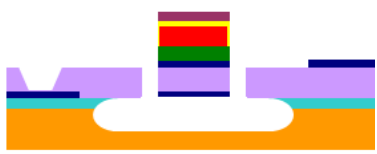

(f)

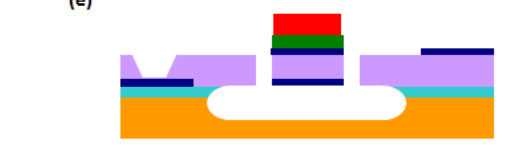

(g)

Figure 3. Fabrication process: a) Sputter deposition of Pt bottom electrode and AlN on Si and LSN; b) Open Via in AlN, lithographic patterning of sputtered $\mathrm{Pt}$ as top electrode, dry etching of $\mathrm{AlN}$ in $\mathrm{Cl}_{2}$ based chemestry; c) Sputter deposition of oxide and evaporation of Iron catalyst; d) SWNTs growth by catalytic CVD at $900{ }^{\circ} \mathrm{C}$ in methane containing atmosphere; e) Spinning of PMGI + Photoresist, development and dry etching of $\mathrm{SiO}_{2}$ in $\mathrm{CF}_{4}$ based chemestry; f) $\mathrm{XeF}_{2}$ dry release of $\mathrm{AlN}$ resonator; g) PMGI + Photoresist removal.

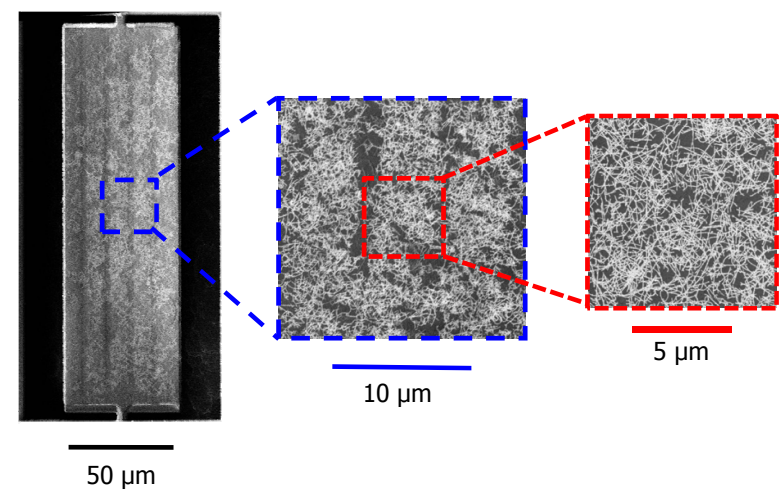

Figure 4. SEM of SWNTs grown on AlN Contour-Mode resonator. The different insets show progressively zoomed in regions of the resonator and demonstrate the quite uniform and dense distribution of SWNTs.

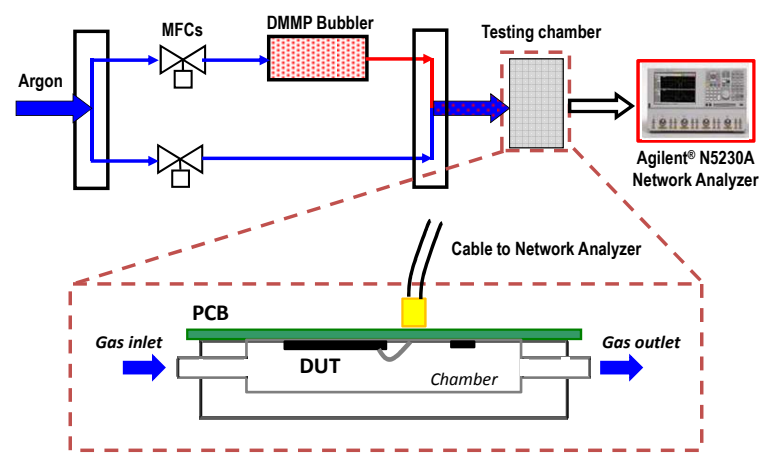

Figure 5. Measurement Setup: the main Argon line is splitted in two indipendently controlled flows. Only one of them carries the analyte (saturated vapor pressure of DMMP). The two flows are recombined before being injected inside the testing chamber. The total flow is always kept constant at $1,000 \mathrm{sccm}$.

\section{A. Measurement Setup}

DMMP is generated by bubbling method using Argon as carrier. Different concentrations of DMMP are produced by mixing DMMP and Argon flows. The die is attached to a custom designed PCB provided with on-board calibration standards and the resonators are wirebonded to $50 \Omega$ lines. Using a voltage controlled single pole six throws RF switch (M/A-COM SW90-0004A) the input power line is first connected to the short, open and load calibration standards and then to one of the wirebonded resonators. As shown in Fig. 5, the PCB itself serves as lid for the testing chamber. The DMMP-induced shifts in resonance frequency are monitored by an Agilent ${ }^{\circledR}$ N5230A Network Analyzer.

\section{B. Sensor Response}

Two different SWNT-functionalized AIN Contour-Mode resonators with resonance frequencies of $287 \mathrm{MHz}$ and $442 \mathrm{MHz}$, respectively, have been tested for DMMP concentrations ranging from 80 to $800 \mathrm{ppm}$. The frequency shifts over time and concentrations of the two devices are shown in Figures 6 and 7, respectively. From these figures it can be observed that the frequency shifts properly increase with DMMP concentration and the response reaches values equal to approximately $80 \%$ of its saturation in less than 1 minute. The frequency drift observed in the response curves 
is due to the limited time given to the devices for recovery (which is achieved solely by flowing Ar). Indeed, for $100 \%$ saturated vapor pressure of DMMP, more than $70 \%$ recovery has been measured in $28 \mathrm{~s}$ and full recovery is achieved in approximately 16 minutes; this proves that the sensor is reversible. In order to demonstrate that the adsorbtion process is due to the presence of SWNTs, a bare resonator without SWNT functionalization layer was exposed to DMMP and showed no detectable changes in its resonance frequency.

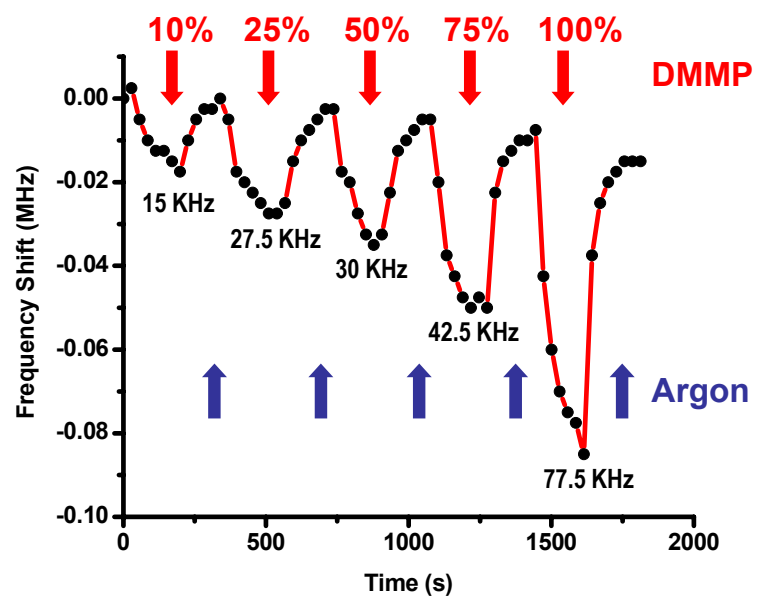

Figure 6. Response to DMMP of a $287 \mathrm{MHz}$ AlN Contour-Mode resonator.

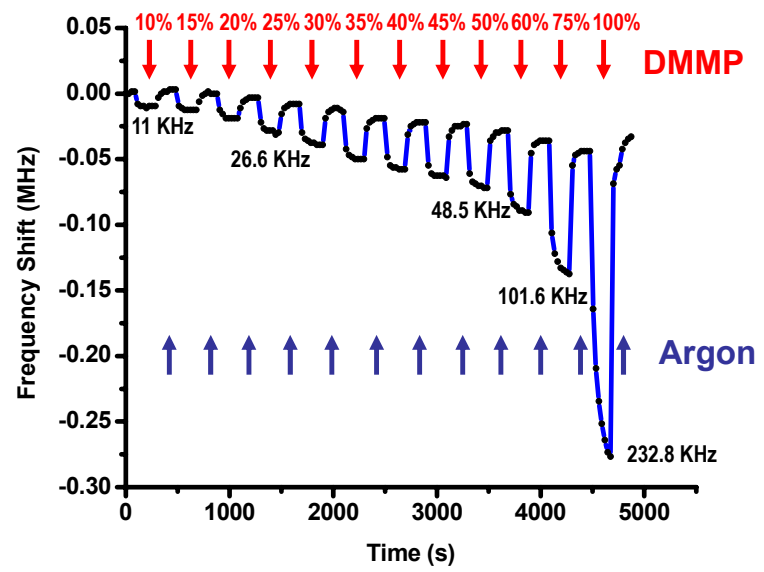

Figure 7. Response to DMMP of a $442 \mathrm{MHz}$ AlN Contour-Mode resonator. The frequency drift is due to the limited time allocated for recovery via $\mathrm{Ar}$ flow. The sensor reaches $70 \%$ recovery in approximately 28 seconds.

\section{Adsorbed mass extraction}

The mass sensitivities of the fabricated devices can be extracted from these measurements by calculating the amount of mass adsorbed on the resonator surface for different concentrations of DMMP. In order to do this, we fit the electrical response of the resonator to the Modified Butterworth van Dyke (MBVD) model. The parallel resistance $R_{0}$ is introduced in order to account for the non infinite resistivity of the Si substrate (these devices were fabricated on low resistivity silicon).

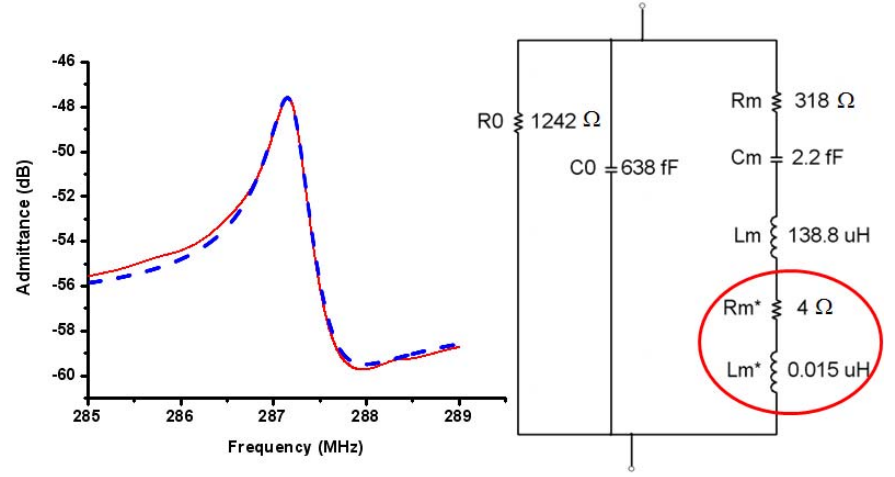

Figure 8. Modified BVD fitting of the resonator admittance curve.

As shown in Fig. 8, the adsorbed mass is modeled by a series resistance, $\mathrm{Rm}^{*}$, which accounts for the additional damping introduced by the adsorption process, and a series motional inductance, $\mathrm{Lm}^{*}$, which is proportional to the adsorbed mass:

$$
\Delta m=2 \cdot n \cdot L m^{*} \cdot \eta^{2}
$$

where $n$ is the number of fingers of the resonator, and $\eta$ is the electromechanical coupling coefficient of the resonator $(\eta$ $\left.=2 \cdot d_{31} \cdot E_{p} \cdot L\right)$. In this way the percentage of mass variation, $\Delta m / m=\Delta L / L$, can be derived and the amount of adsorbed mass is found by multiplying this ratio for the unloaded mass of the resonator. The resonator mass is calculated, in turn, from the geometrical dimensions and mass density of the fabricated device.

The calculated values of adsorbed mass are plotted versus the relative concentrations of DMMP in Fig. 9. The experimental data points can be fitted by a BET isotherm curve [1]. This fitting highlights how these sensors undergo a multilayer adsorptions process for high concentrations of DMMP.

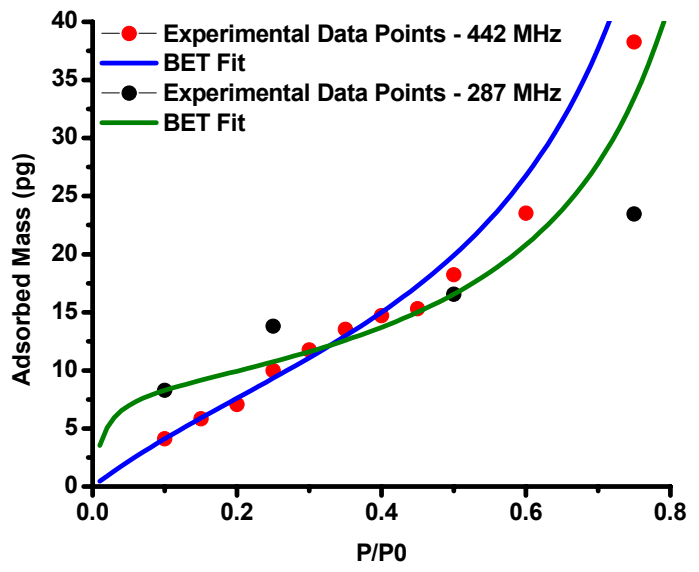

Figure 9. Adsorbing isotherms for the two AIN Contour-Mode Resonators of this work.

Finally, dividing the resonance frequency shift by the correspondent value of adsorbed mass, the mass sensitivity of the device is calculated. In Table III, the experimental values of mass sensitivity for the fabricated devices are compared 
with the analytical values calculated as shown in section II. These data show a very good agreement between theory and experiments and further confirm a clear understanding of the sensor operations.

TABLE III. MASS SENSITIVITIES FOR THE FABRICATED DEVICES

\begin{tabular}{c|c|c} 
Mass Sensitivity & $\mathbf{2 8 7} \mathbf{M H z}$ & $\mathbf{4 4 2} \mathbf{M H z}$ \\
\hline Experimental & $1.8 \mathrm{KHz} / \mathrm{pg}$ & $2.65 \mathrm{KHz} / \mathrm{pg}$ \\
\hline Analytical & $1.8 \mathrm{KHz} / \mathrm{pg}$ & $2.65 \mathrm{KHz} / \mathrm{pg}$
\end{tabular}

\section{CONCLUSION}

Design, fabrication and testing of a novel gravimetric sensor based on the direct integration of SWNTs on AlN contour-mode resonator have been demonstrated. The sensor design has been optimized in order to maximize the sensitivity without affecting the resonator performance or impacting its geometry. Two different levels of mass sensitivity equal to $1.8 \mathrm{KHz} / \mathrm{pg}$ and $2.65 \mathrm{KHz} / \mathrm{pg}$ have been measured on the same chip. The demonstrated performance of AlN contour-mode resonators as gravimetric sensors combined with the sensing properties of SWNTs and the capability to functionalize them in order to make them selective to specific analytes make this new technology suitable for the fabrication of compact, low cost and high performance sensors platform for multiple VOC detection.

\section{ACKNOWLEDGMENT}

This work was supported by NCMR and NSF. The authors wish to thank the staff at The University of Pennsylvania Wolf Nanofabrication Facility (WNF).

\section{REFERENCES}

[1] D.S. Ballantine et al., "Acoustic wave sensors-Theory,Design and Physico-Chemical Applications”, Academic Press, 1996.

[2] M. Li, H. X. Tang and M. L. Roukes, "Ultra-sensitive NEMS-based cantilever for sensing, scanned probe and very high ", Nature Nanotechnology 2, 114 - 120 (2006)

[3] M. Penza et al. "TFBAR gas sensor functionalized with nanocomposite LB layer of CNTs ", IEEE trans. on Electron Device, Vol.55, No.5, May 2008.

[4] G. Sauerbrey, "Use of the vibrating quartz for thin film weighing and microweighing", (in German) "Verwendung von Schwingquarzen zur Wagung dunner Schichten und zur Mikrowagung", Z. Phys., 155 (1959) 206-222

[5] J. R. Vig, and F. L. Walls, "A review of sensor sensitivity and stability", IEEE International Frequency Control Symposium, 2000.

[6] G. Piazza, P. J. Stephanou, A. Pisano, "One and two port piezoelectric higher order contour-mode MEMS resonator for mechanical signal processing”, Solid State Electronic, Vol. 51, Issues 11-12, Dec.2007, pages 1596-1608.

[7] R. Abdolvand, Z. Hao, F. Ayazi, "A Temperature Compensated Zn-O Diamond Resonant Mass Sensor", IEEE Sensors 2006.

[8] W. Pang et al. "Femtogram Mass Sensing Platform based on Lateral Extensional Mode Piezoelectric Resonator", Applied Physic Letter 88, 243503, 2007.

[9] D. E. Johnston, M. F. Islam, A. G. Yodh and A. T. Johnson, "Electronic device based on purified carbon nanotubes grown by high-pressure decomposition of carbon monoxide", Nature Materials, Vol.4, August 2005.

[10] C. Y. Lee and M.Y. Strano, "Understanding the dynamics of signal transduction for adsorption of gases and vapors on carbon nanotubes sensors", Langmuir 2005, 21, 5192-5196

[11] G. Piazza, P.J. Stephanou, A.P. Pisano, "Single-Chip MultipleFrequency AIN MEMS Filters Based on Contour-Mode Piezoelectric Resonators", Journal of MicroElectroMechanical Systems, vol. 16, no.2, pp. 319-328, April 2007.

[12] C. Staii, A.T. Johnson, M. Chen, A. Gelperin, "DNA-decorated carbon nanotubes for chemical sensing.", Nano Lett. 5, 1774-1778 (2005).

[13] N.R. Franklin, Y. Li, R.J. Chen, "Patterned growth of single-walled carbon nanotubes on full 4-inch wafer", App. Phys. Lett., Vol.79, No.27, 2007 